MATEC Web of Conferences 12, 05006 (2014)

DOI: $10.1051 /$ matecconf $/ 20141205006$

(C) Owned by the authors, published by EDP Sciences, 2014

\title{
Transferability of fatigue resistance data for welded joints
}

\author{
Matthias Kaffenberger ${ }^{1, a}$, Ina Platte ${ }^{2}$, Ehsan Shams² and Michael Vormwald ${ }^{2}$ \\ ${ }^{1}$ TU Darmstadt, Institue for Materials Technologie, 64283 Darmstadt, Germany \\ 2 TU Darmstadt, Materials Mechanics Group, 64287 Darmstadt, Germany
}

\begin{abstract}
The original notch stress approach for fatigue design is limited to thick sheet structures and a fixed notch radius of $1 \mathrm{~mm}$. Concepts utilizing the effective notch stress claim to account for the variation of the weld shape parameters, however, size effects are not taken into account. Therefore, an expansion of the notch stress approach has been developed, which includes consideration of the geometrical and statistical size effect in order to extend the application to thin and thick sheet structures. It is also capable of evaluating highly localized three-dimensional stress concentrations at weld start and end points. To validate the quality of this approach, experimentally determined fatigue data for thin steel sheet specimens from the Institute of Material Mechanics, including weld start and end points, and data from the literature regarding conventional thick sheet components without weld start and end points were reevaluated. The final result is a unified scatter band of fatigue lives of thin and thick sheet specimens, with and without weld start and end points, including also several modes of seam weld end point loading: transversal and longitudinal tension compression as well as shear loading at seam welds.
\end{abstract}

\section{Introduction}

The fatigue life of welded structures is often determined using S-N (Wöhler) curves. The most common parameters to describe the rupture of specimens are stresses. Today, a variety of different Wöhler curves for welded structures exist. If local concepts - like the notch stress concept - are used, even under consideration of different size effects, standardisation of Wöhler curves is hardly possible.

The major aim of this research was to unify structures different in size and shape under one scatter band. Therefore, a "Numerical Method regarding Size Effects for Standardisation of Wöhler Curves" (NuMeSiS) was developed.

\section{The approach "NuMeSiS"}

As a basic assumption, size effects are responsible for the big differences in S-N curve parameters for welded structures. Therefore, the geometrical and statistical size effect will be taken into account in a completely new way. In step 1 (see Fig. 1 left), for each point on the surface of the weld notch, the effective stress according to Neuber [1] will be calculated which leads to an effective stress distribution

\footnotetext{
${ }^{a}$ Corresponding author: kaffenberger@mpa-ifw.tu-darmstadt.de
}

This is an Open Access article distributed under the terms of the Creative Commons Attribution License 4.0, which permits unrestricted use, distribution, and reproduction in any medium, provided the original work is properly cited. 


\section{MATEC Web of Conferences}

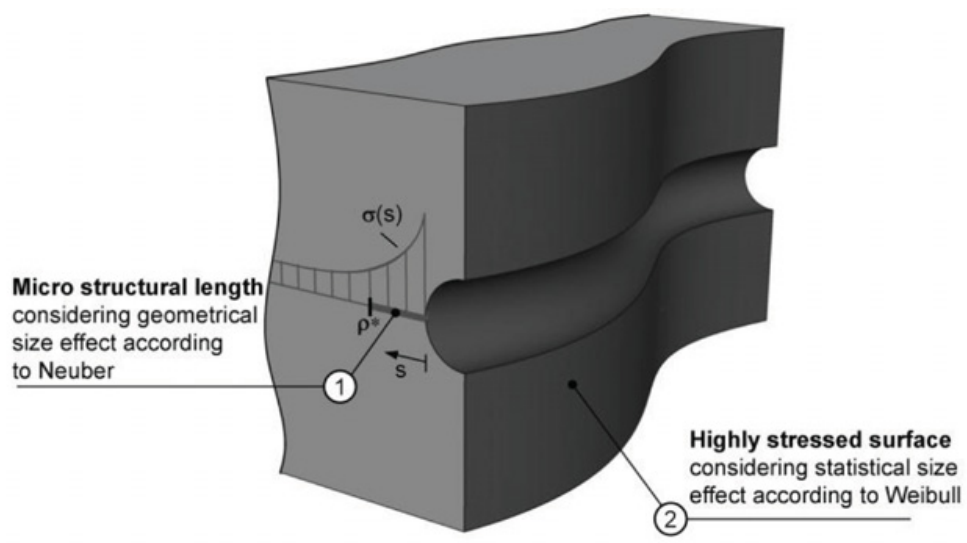

Figure 1. Steps of the Method NuMeSiS.

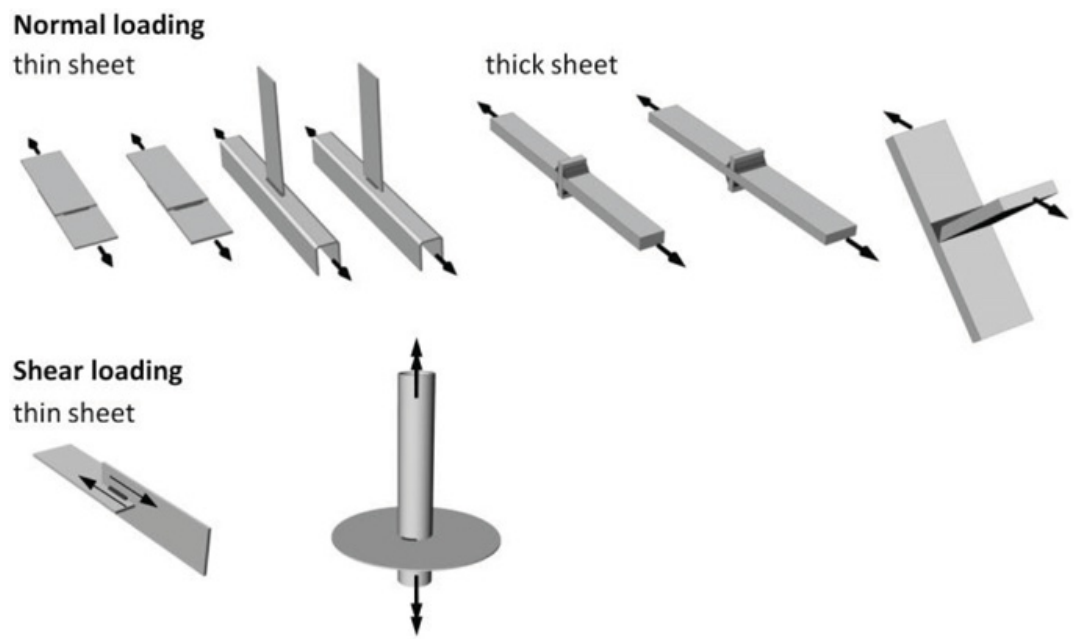

Figure 2. Specimen overview.

which takes the geometrical size effect into account. In step 2 (see Fig. 1 right) the highly stressed surface according to Weibull [2] will be calculated based on the effective stress distribution according to Neuber. The result is a highly stressed surface layer, which takes the geometrical and statistical size effect into account at the same time. The resulting stress is the weighted effective von Mises stress $\sigma_{\rho *, \kappa}$, where $\rho^{*}$ is the micro structural length and $\kappa$ the Weibull exponent. Thus, the results a nearly independent from the modelled notch radius.

To be able to calculate stress along the micro structural length and the highly stressed surface via Finite Element Method the approach "SPIEL" by Diemar [3] was modified. As an additional boundary condition case, all surface nodes will be supported in all directions. This additional calculation step is loaded with a unified pressure $\mathrm{p}=1$. The vector of the reaction forces is equal to the mean normal direction of the surface node. Along this direction the effective stress is calculated. The magnitude of the reaction force is equal to the according surface for each node from which the highly stressed surface can be derived. 


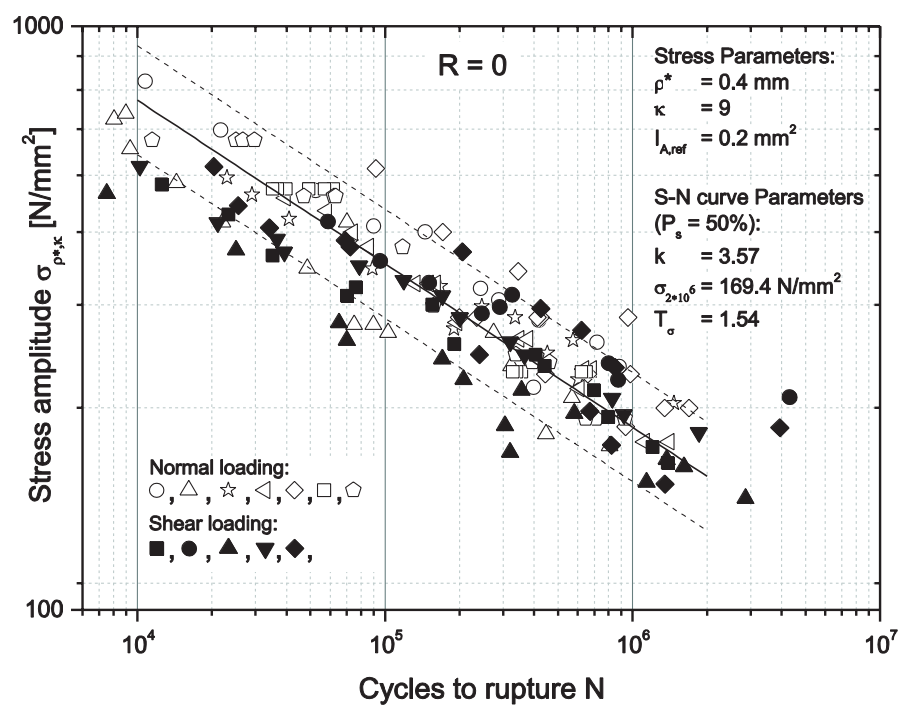

Figure 3. Standardised S-N Curve for weighted effective Stress.

\section{Results and conclusion}

The parameters $\rho^{*}$ and $\kappa$ were determined from thin and thick sheet specimens under normal and bend loading. To validate these results thin sheet specimens under shear load were tested. An overview over the specimens is given in Fig. 2.

The thin sheet overlap joints and T-jonts vary in sheet thickness and weld length which leads to a total of 7 different test series under normal and bend loading including thick shett structures. The thin sheet overlap joints under shear loading vary in weld thickness and weld length. Also a tubular joint was tested which leads to total of five different test series under shear loading.

After applying the Method NuMeSiS to all test series, the scatter band in Fig. 3 was obtained. All test series were modelled with their actual notch radius. By considering the geometrical and statistical size effect dependently it was possible to unify all 12 test series (sheet thickness 2 to $15 \mathrm{~mm}$, notch radius 0.2 to $1.5 \mathrm{~mm}$ ) under one scatter band. The difference in the slope of the S-N curve regarding normal and shear loading still can be seen, but a strict distinction of both loading cases is not necessary.

The biggest achievement however, is that it is no longer necessary to know the notch radius in advance. The consideration of size effects in this ways dampens the modelling influence to very little significance. The only parameters needed are the micro structural length, the Weibull exponent and a reference surface.

\section{References}

[1] Neuber, H.: Über die Berücksichtigung der Spannungskonzentration bei Festigkeitsberechnungen, Konstruktion 20 Heft 7, 1968, 245-251

[2] Weibull, W.: A statistical distribution function of wide applicability, Journal of Applied Mechanics 18, 1951, 293-297

[3] Diemar, A., Thumser, R., Bergmann, J.W.: Statistischer Größeneinfluss und Bauteilfestigkeit. Eine neue Methode zur Ermittlung von Spannungsintegralen, Materialprüfung 46, 2004, 16-21 